Graduate Students, Energy and Environment

\title{
Self-Healing Performance of Smart Polymeric Coatings Modified with Tung Oil and Linalyl Acetate
}

Norhan Ashraf Ismail 1,2, Adnan Khan 1, Ramazan Kahraman 2,*and Rana Abdul Shakoor 1,*

${ }^{1}$ Center for Advanced Materials, Qatar University, Doha 2713, Qatar; ni1300925@qu.edu.qa (N.A.I.); ak1704740@qu.edu.qa (A.K.)

2Department of Chemical Engineering, College of Engineering, Qatar University, Doha 2713, Qatar

*Correspondence: ramazank@qu.edu.qa (R.K.); shakoor@qu.edu.qa (R.A.S.);Tel.: +974-440-341-30 (R.K.);

+974-440-368-67 (R.A.S.)

\section{Abstract}

This work focuses on the synthesis and characterization of polymeric smart self-healing coatings. A comparison of structural, thermal, and self-healing properties of two different polymeric coatings comprising distinct self-healing agents (tung oil and linalyl acetate) is studied to elucidate the role of self-healing agents in corrosion protection. Towards this direction, urea-formaldehyde microcapsules (UFMCs) loaded with tung oil (TMMCs) and linalyl acetate (LMMCs) were synthesized using the in-situ polymerization method. The synthesis of both LMMCs and TMMCs under identical experimental conditions $\left(900 \mathrm{rpm}, 55^{\circ} \mathrm{C}\right)$ has resulted in a similar average particle size range $(63-125 \mu \mathrm{m})$. The polymeric smart self-healing coatings were developed by reinforcing a polymeric matrix separately with a fixed amount of LMMCs ( $3 \mathrm{wt} . \%$ and $5 \mathrm{wt} . \%$ ), and TMMCs (3 wt.\% and 5 wt.\%) referred to as LMCOATs and TMCOATs, respectively. The development of smart coatings (LMCOATs and TMCOATs) contributes to achieving decent thermal stability up to $450{ }^{\circ} \mathrm{C}$. Electrochemical impedance spectroscopy (EIS) analysis indicates that the corrosion resistance of smart coatings increases with increasing concentration of the microcapsules (TMMCs, LMMCs) in the epoxy matrix reaching $\sim \mathrm{G} \Omega$. As a comparison, LMCOATs containing 5 wt.\% LMMCs demonstrate the best stability in the barrier properties than other developed coatings and can be considered for many potential applications. 7.

\section{Experimental}

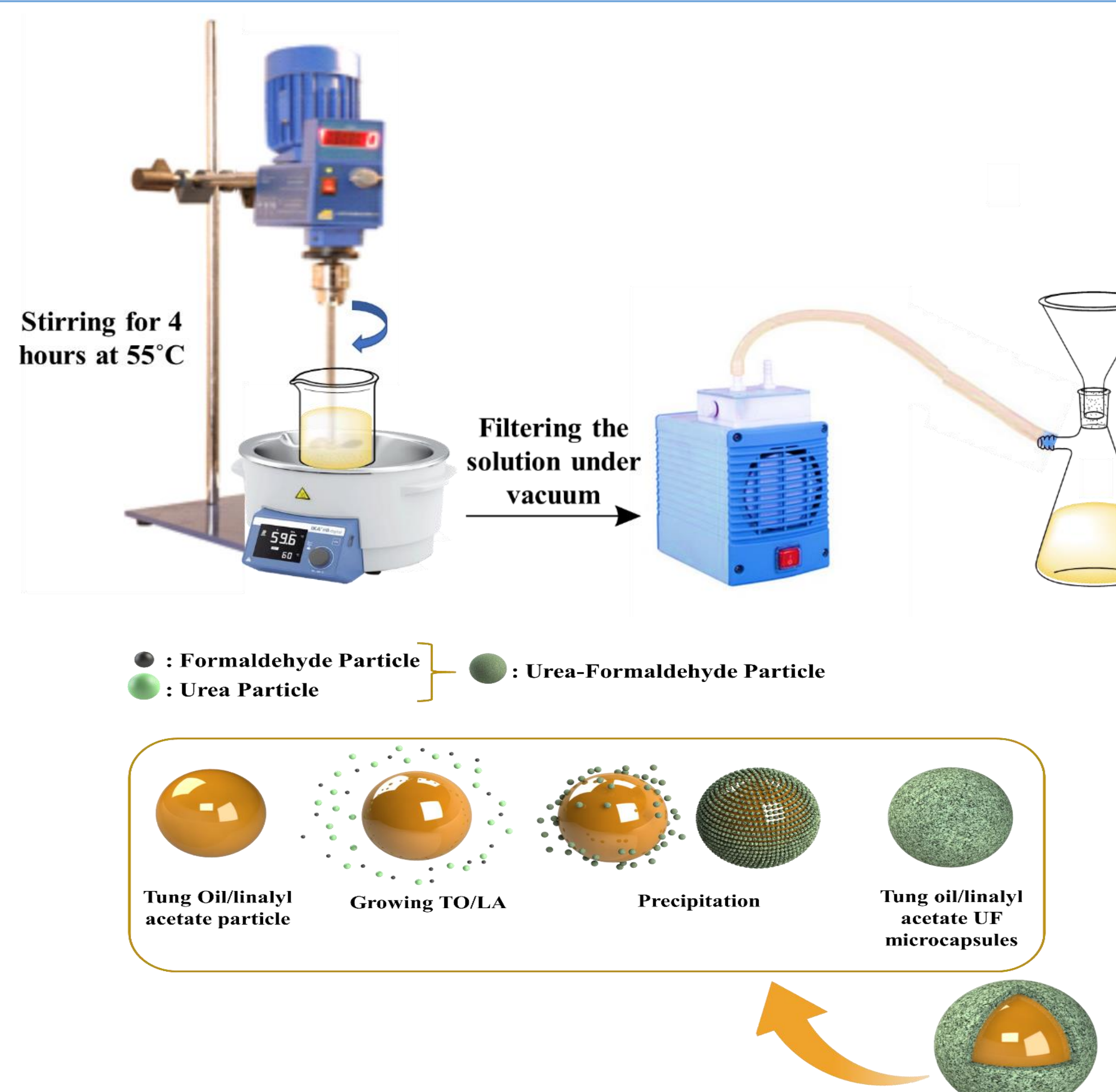

\section{Preparation of Coatings}

- Coatings containing LMMCs and TMMCs were prepared using 3.0 $\mathrm{wt} \%$ and $5.0 \mathrm{wt} \%$ of microcapsules for comparison.

-The coatings were subjected to a controlled damage, self healing and corrosion resistance was analyzed after different time interval.

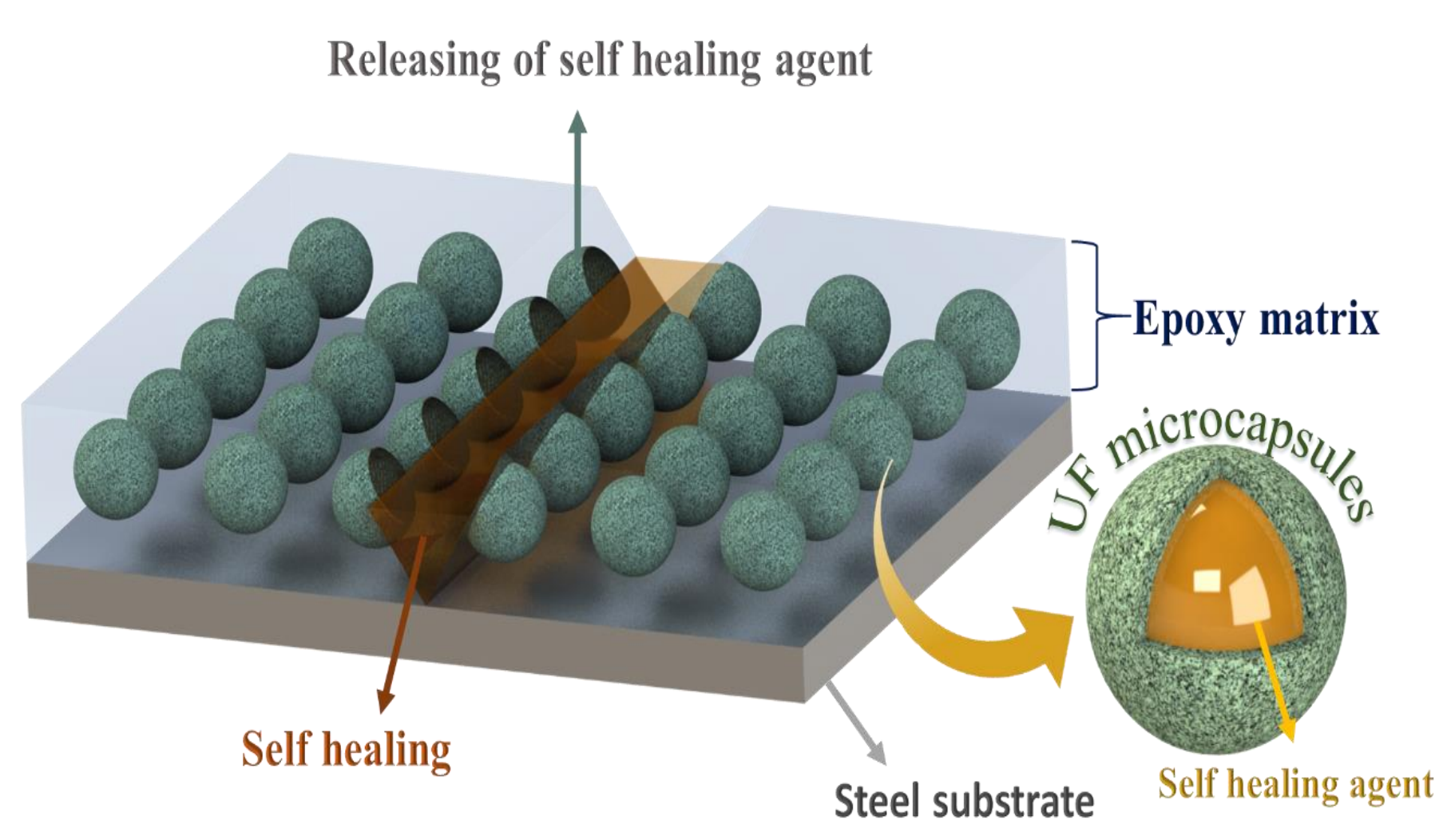

Schematic diagrams of smart coatings

\section{Result and Discussion}
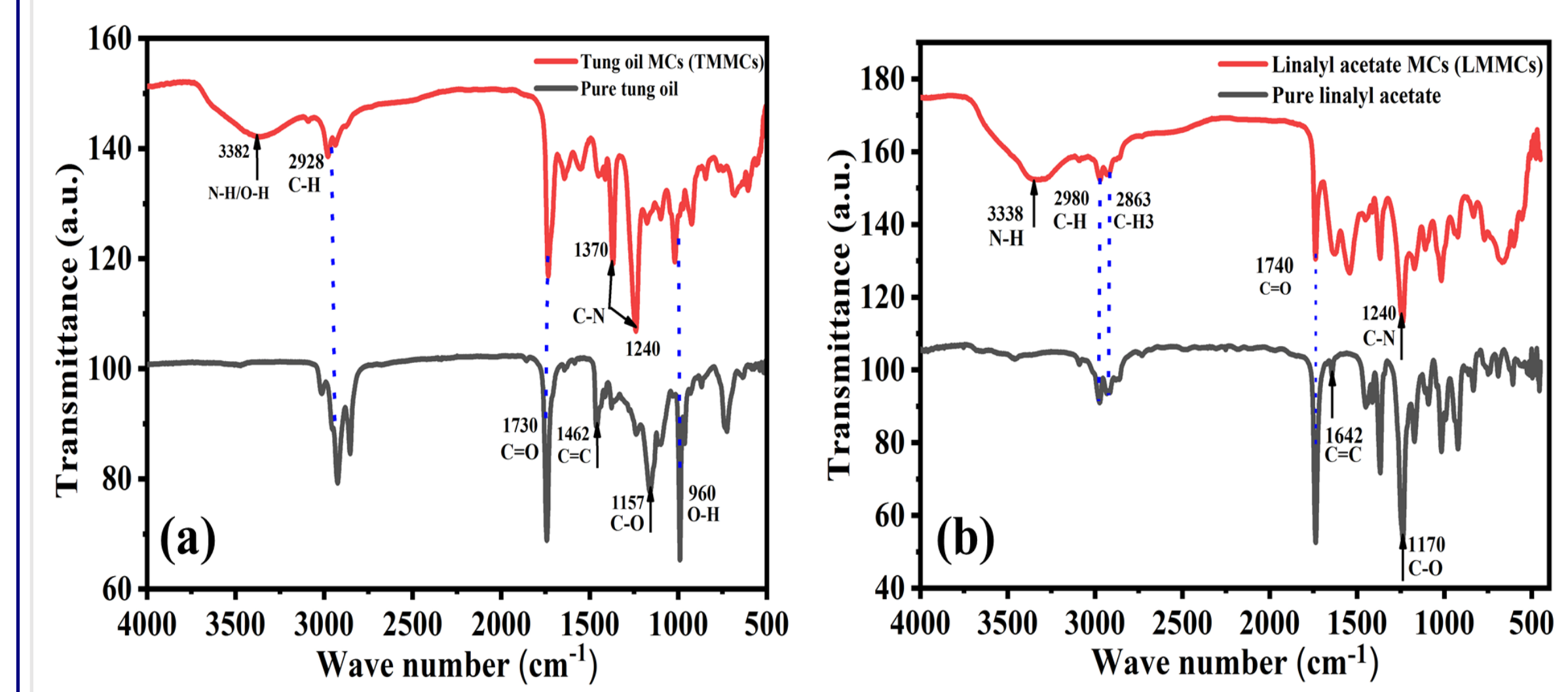

Fig. 1: FTIR spectra of (a) pure linalyl acetate and LMMCs (b) pure tung oil and TMMCs

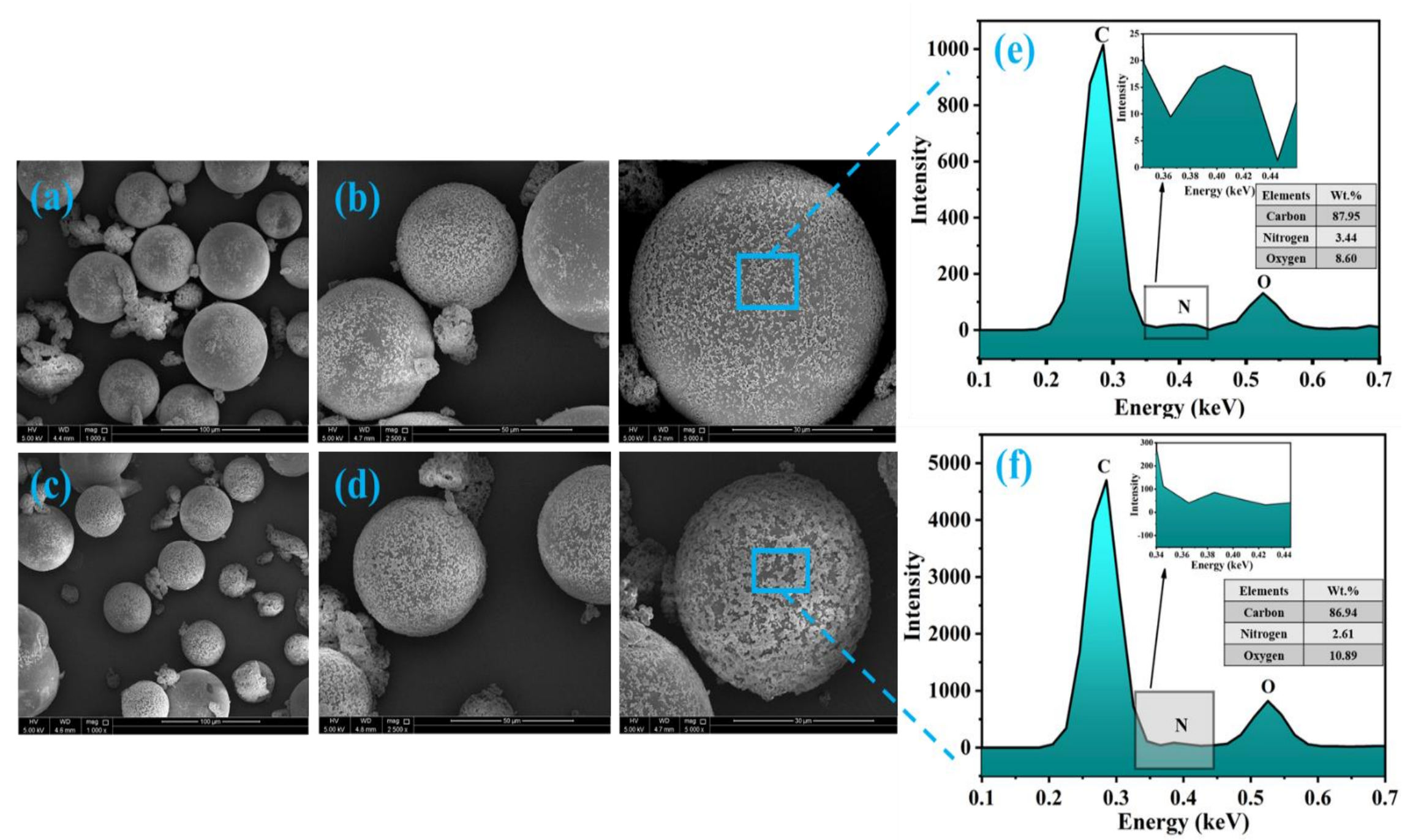

Fig. 2: Surface morphology of the microcapsules at different resolution $(a, b)$ urea-formaldehyde microcapsules loaded with linalyl acetate-LMMCs (c, d) urea-formaldehyde microcapsules loaded tung oil-TMMCs d (e, f) EDX analysis of LMMCs and TMMCs.

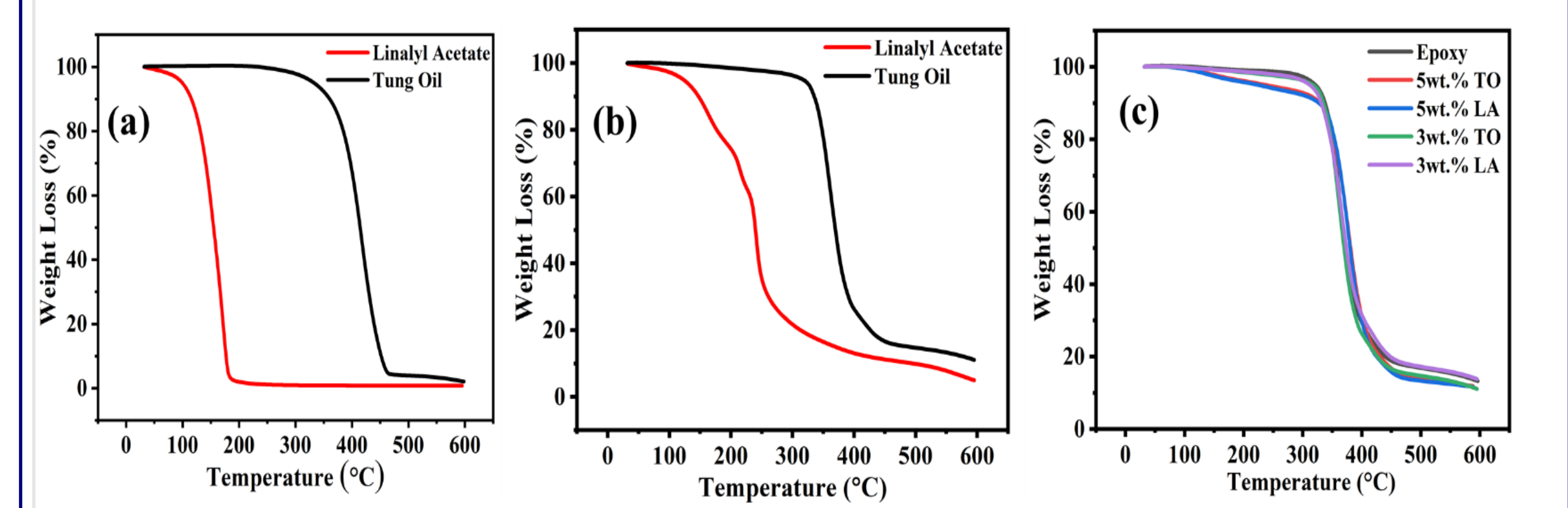

Fig.3: TGA results; (a) pure LA and TO, (b)urea-formaldehyde microcapsules loaded with linalyl acetate-LMMCs and ureaformaldehyde microcapsules loaded with tung oil-TMMCs, (c) Reference coatings and TMCOATs and LMCOATs.

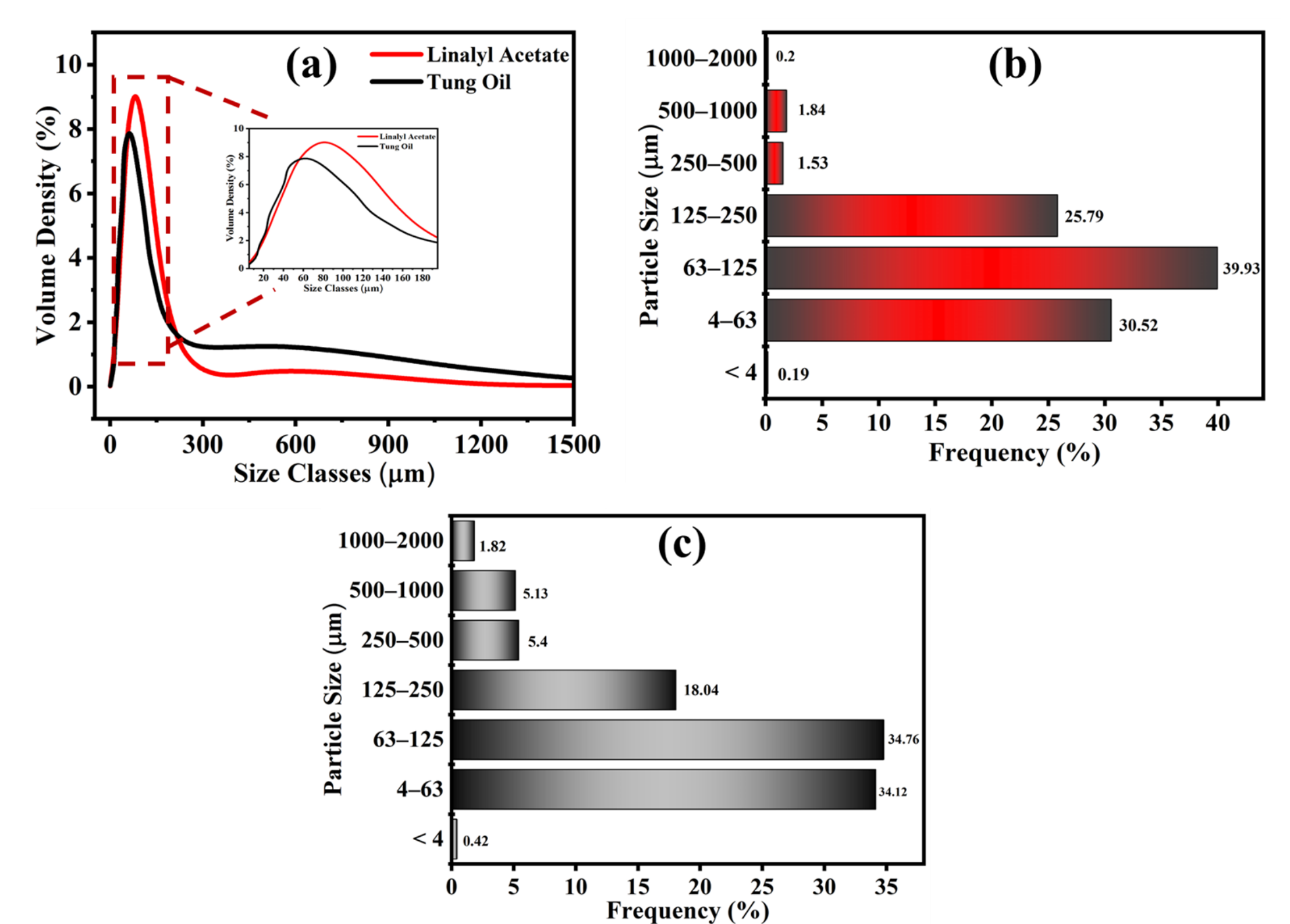

Fig. 4: Particle size analysis. (a) Gaussian particle size distribution in LMMCs and TMMCs, (b) bar chart showing distribution of LMMCs and (c) bar chart showing distribution of TMMCs.

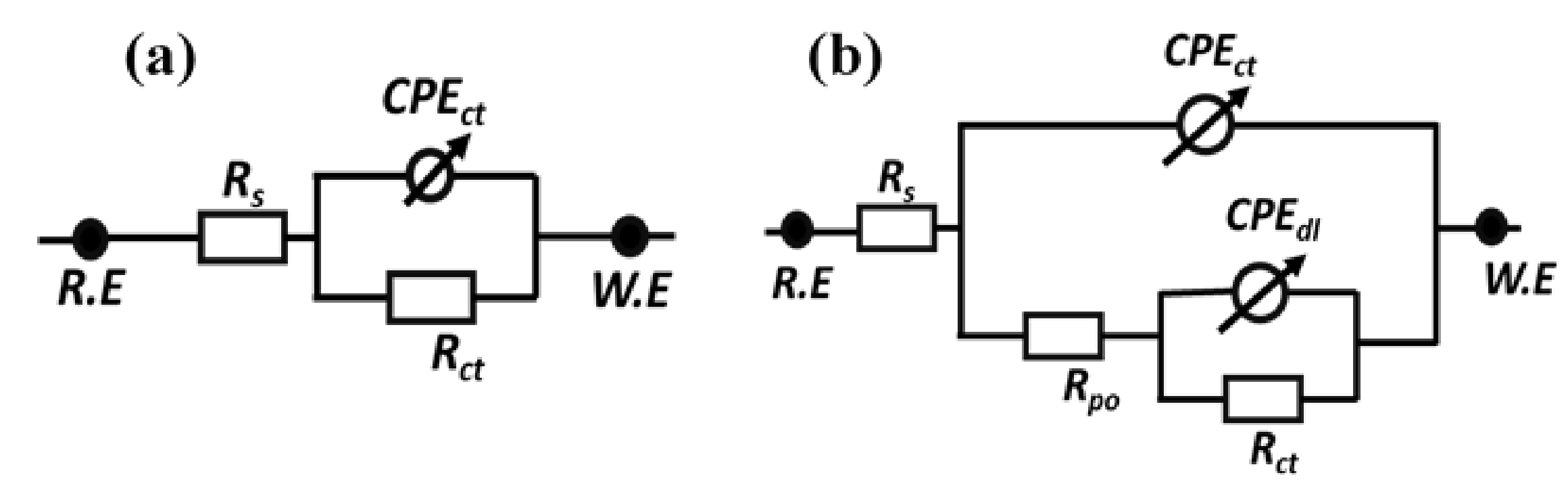

Fig. 5: Equivalent circuits used to fit the electrochemical impedance spectroscopy data (a) reference coatings (b) TMCOATs and LMCOATs.

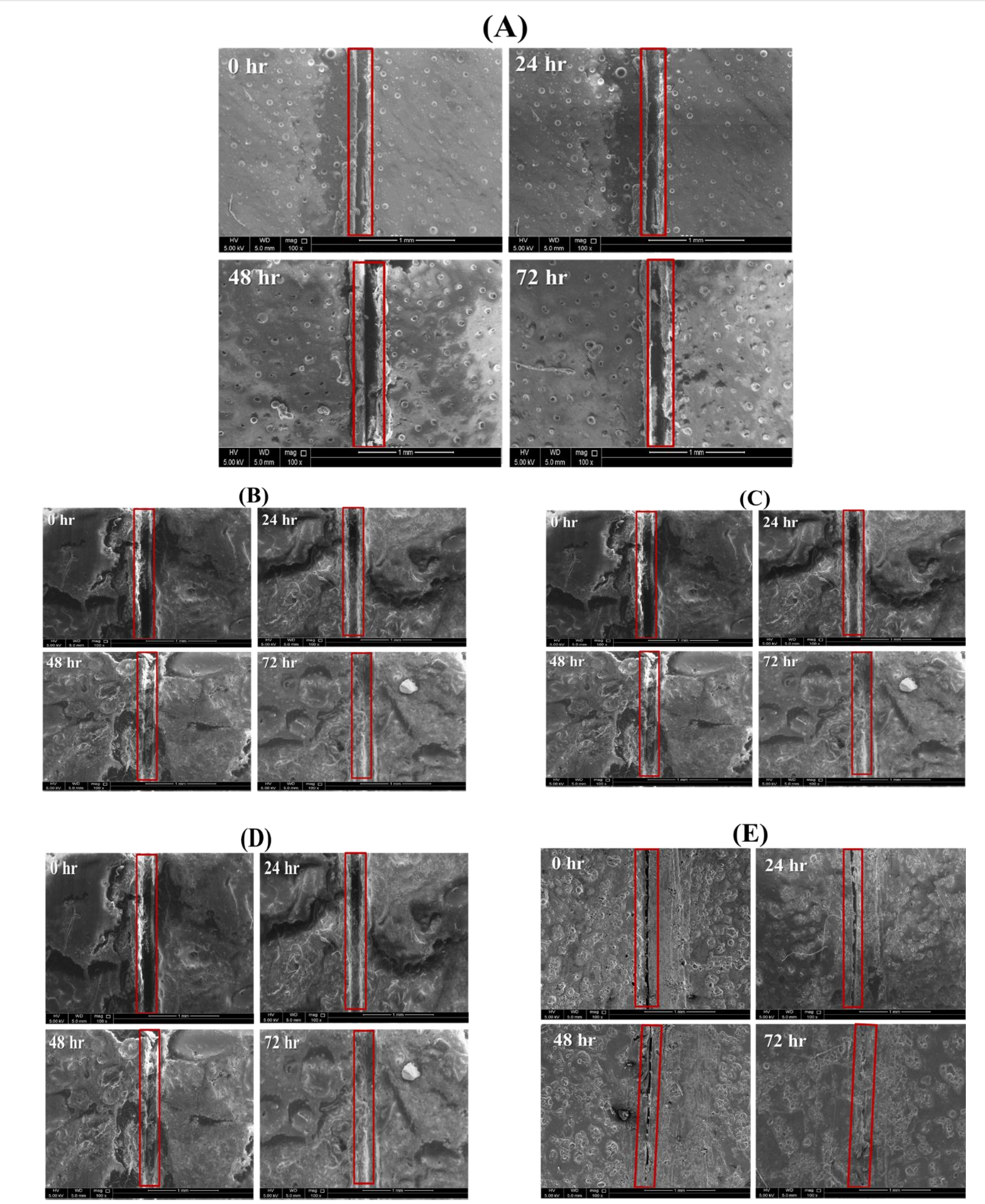

Fig. 6: Self-healing analysis of smart coatings at various time intervals, (A) Reference coating (plain epoxy), (B) $3 \mathrm{wt} \%$ TMCOATs, (C) $3 \mathrm{wt} \%$ LMCOATs, (D) $5 \mathrm{wt} \%$ LMCOATs and (E) $5 \mathrm{wt} \%$ TMCOATs
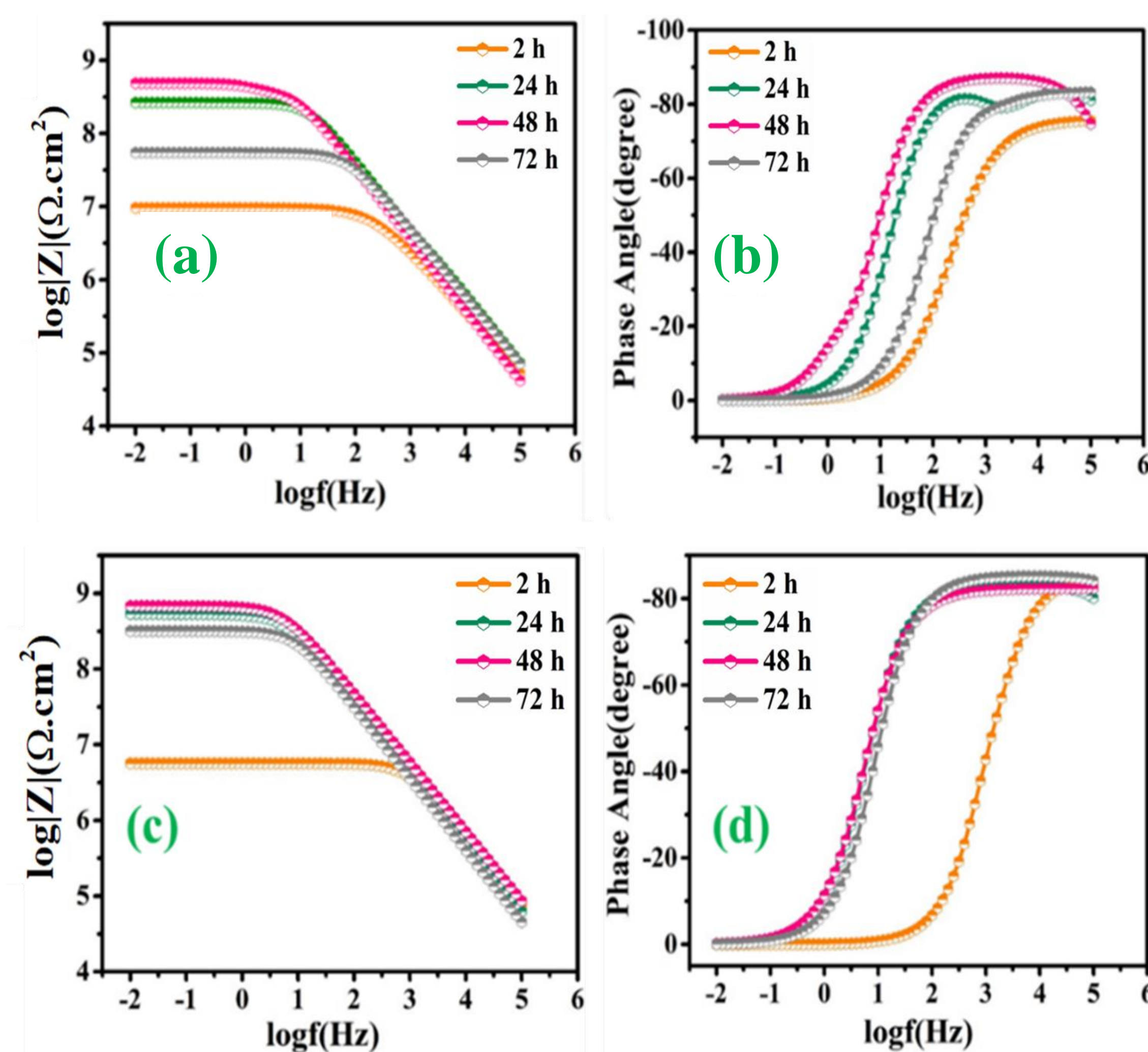

Fig. 7: (a) Bode graph of 5 wt.\% TMMCs (b) phase angle graph $5 \mathrm{wt} . \%$ TMMCs, (c) Bode graph of $5 \mathrm{wt} \%$ LMMCs, (d) phase angle graph $5 \mathrm{wt} . \%$ LMMCs.

\section{Conclusion}

Self-healing smart coatings (TMCOATs and LMCOATs) containing $3 \mathrm{wt} . \%$ and $5 \mathrm{wt} . \%$ TMMCs and LMMCs were respectively developed and characterized.

The developed smart coatings demonstrate superior corrosion resistance when compared to the reference coatings.

The self-healing characteristics of smart coating are sensitive to the concentration of TMMCs and LMMCs. Comparatively, LMCOATs exhibit more stable barrier properties reaching $\sim 1 \mathrm{Gohm}$, when compared with other smart coatings depicting their usefulness for some industrial applications.

\section{Acknowledgment}

This publication was achieved and published with the support of the NPRP Grant (NPRP11S-1226-170132)

from Qatar National Research Fund (QNRF) (a member of the Qatar Foundation). 